\title{
Risk factors for infection following primary total knee replacement.
}

\author{
Linjie Hao, Yumin Zhang*, Wei Song, Tao Ma, Jun Wang, Huiguang Cheng, Kun Li, Siqing Qin \\ Joint Surgery Department, Hip Ward, Hong-Hui Hospital, Xi'an Jiaotong University College of Medicine, Xi'an City, \\ Shaanxi Province, PR China
}

\begin{abstract}
Purpose: Information about risk factors can be used to target preventive measure on susceptible patient subgroups. The purpose of study was to determine the risk factors for infection following primary knee replacement.

Methods: Between April 2014 and January 2016, total 1599 primary total knee replacements were carried out in 1374 patients, among them 1161 (933 female and 228 male) cases were available for final study. Patients were divided into without infection and had deep infection. Patients-related risk factors and provider-related risk factors were determined.

Results: Out of 1161 patients, 16 patients had deep infection with infection rate was $1.38 \%$. There was no significant statistically between age, diagnosis, obesity and malnutrition with infection. Total $16.6 \%$ were smoker, among infected group 37.5\% patients were smoker with odds ratio 3.01 and $P$ value 0.013 $(<0.05)$. In study $12.4 \%$ patients had diabetics mellitus, in infected group $25 \%$ had with odds ratio 2.35 and $p$ value $0.016(<0.05)$. Total $2.6 \%$ patients had history of steroid use, among infected group $12.5 \%$ patients with odds ratio 5.39 and $p$ value less than 0.05 . Regarding the provider-related risk factors, mean duration of surgery was $\mathbf{1 3 0 . 9}$ min and mean duration of hospital stay was 10.95 days with both had no significant statistically. Mean blood loss during surgery was $751.47 \mathrm{ml}$ and mean amount of blood transfusion was $596.08 \mathrm{ml}$ with both had association with infection $(\mathrm{p}<0.05)$.

Conclusions: Smoking, diabetes mellitus, steroid use, total blood loss and blood transfusion were significance risk factors for infection after total knee replacement.
\end{abstract}

Keywords: Risk factors, Infection, Primary total knee replacement.

Accepted on November 16, 2017

\section{Introduction}

Total knee replacements are widely recognized as being successful treatment and are now among the most common major surgical procedure, annual numbers of operations have been increasing continuously. TKRs improve functional status, relieve pain results in relatively low postoperative morbidity. Additionally, the results of surgery are durable with 10-15 y implant survival [1,2]. The incidence of failure after knee replacement is low. Nonetheless, some patients achieve a poor result after surgery or the implant fails prematurely and revision operation is required. The most common indications for revision are infection, loosening, instability, fracture and patella-femoral complications.

Post-operative infection in TKR is one of most divesting and challenging complication which has adverse effects on health and functional status of the patients and use up valuable health care resources. Despite continue efforts to prevent postoperative infections, TKR infection complicated up to $1 \%$ $[3,4]$. At present removal of infected prosthesis is usually necessary to control the infection. Repetitive surgeries, prolonged hospitalization and rehabilitation leads to decline in quality of life and possibly compromised function even in the longer term making TKR infection a catastrophic event for a patient. For the same reasons TKR infection causes a considerable burden on society.

Until now the decline in the TKR infection rate has been achieved by improving the operative environment and surgical techniques and by introducing antibiotic prophylaxis. Minimal invasive surgery, novel ways to administer antibiotic locally (such as biodegradable polymers) and prosthesis with antibacterial surface coating are potential ways to further reduce the rates of infection. The use of such special techniques is, however, limited to certain patient subgroups and in general supplementary approaches are needed. It is important to identify and investigate risk factors for knee infection. Multiple risk factors for TKR infection have been identified. It is important to know about patients' general health and co-morbid conditions preoperatively to control infection. It is hypothesis that if patients general characteristics and co-morbid condition can improve preoperatively, postoperative TKR infection can also control. 


\section{Materials and Methods}

\section{Study design}

This is retrospective study. Study was conducted in the department of joint surgery of a hospital. Data was obtained retrospectively.

\section{Data collection}

Data was retrieved from the Record/Book-keeping section and computerized record database system "Hospital Info Sys" of the hospital.

Clinical data was entered retrospectively at each follow-up after surgery.

Full medical records and diagnostic tests and radio graphs were reviewed if needed by surgeons.

\section{Study population}

From April 2014 to January 2016, total of 1599 primary total knee replacement surgeries were performed in 1374 patients in Xi' an Honghui hospital. Among them 42 patients died and 171 patients lost follow up. Total 1161 patients were available for final study. Sixteen patients got infection after primary TKR, seven were male and nine were female.

\section{Inclusion criteria}

All patients with primary total knee replacement surgery performed from $1^{\text {st }}$ April 2014 to $1^{\text {st }}$ January 2016 were included. Patients were included regardless of their diagnosis. Patients were included with deep infection.

\section{Exclusion criteria}

All revision knee replacements were excluded.

Surgery performed outside the alleged period and place were excluded. Patients who had suture site infection were also excluded.

The patients who lost in follow up and patients who died after primary TKR

\section{Follow-up}

Postoperative follow up was done at $0.5-2.5 \mathrm{y}$ with mean $1.6 \mathrm{y}$ at irregular intervals depending on their clinical status and consulting surgeon's suggestions.

\section{Measurements}

Full medical records and clinical presentations were reviewed. Patients' age at operation, gender, diagnosis, side of TKR done noted. Smoking history, history of steroid use taken by patients and recorded data. Presence of DM and HTN taken from recorded data and conformed by patients. Obesity was calculated according to BMI of patients and categorized accordingly WHO. Malnutrition was calculated according to
BMI, total lymphocyte count, serum albumin level. Duration of surgery, hospital stay, total blood loss and blood transfusion were taken from recorded data.

\section{Data analysis}

Data were analysed with SPSS version 20.0 (SPSS Inc., Chicago IL). Descriptive analysis by means, standard deviation was used for continuous variables. A non-parametric, MannWhitney $U$ test was used for group analysis on continuous variables. Categorical variables in groups were analysed by Multi-way ANOVA and odds ratio. A p value of $<0.05$ was regarded as statistically significant.

\section{Results}

Between April 2014 and January 2016, total 1599 primary Total Knee Replacements (TKRs) were carried out in 1374 patients. Among them 171 had been lost to follow-up and 42 patients died. In total 1161 patients were available for final study. Out of 1161,16 patients had infection (1.38\%). The study was correlated and analysed with their intrinsic factors (patient-related) and extrinsic factors (provider-related) with infection.

\section{Patient related factors}

Age: The minimum age of patient in study group is $32 \mathrm{y}$ male which was diagnosed for ankylosing spondylitis and had TKR; maximum age is $84 \mathrm{y}$ female with osteoarthritis. The mean age of patient was $64.37 \mathrm{y}$. Among non-infected group mean age was $64.44 \mathrm{y}$ and infected group it was $62.75 \mathrm{y}$. There was no significant statistical variation in age of between infected and non-infected groups as p value is $0.704(>0.05$, Table 1$)$.

Table 1. Showing mean, median and standard deviation of age between two groups.

\begin{tabular}{llllll}
\hline & Number & Mean $(\mathbf{y})$ & Median $(\mathbf{y})$ & SD & p value \\
\hline Infection & 16 & 62.75 & 64.00 & 6.50 & 0.704 \\
\hline $\begin{array}{l}\text { No } \\
\text { infection }\end{array}$ & 1145 & 64.44 & 64.00 & 8.78 & \\
\hline
\end{tabular}

Age distribution by box plot between these two groups is showing below. Most of the patients were between ages 55-70 y (Figure 1).

Gender: Among total 1161 patients, 933 (80.39\%) were female and $228(19.61 \%)$ were male with the ratio of $4.1: 1$ (Figure 2).

Among them sixteen had infection, nine were female and seven were male, with nearly $50 \%$ each. Chi square test was performed to compare gender variation between infection and non-infection group. There is no significantly statistical difference between groups with $\mathrm{p}$ value was $0.118(>0.05)$.

Diagnosis: Regarding the diagnosis, majority of patients had TKR for Osteoarthritis which accounts for $95.1 \%$ then comes for Rheumatoid arthritis with $2.9 \%$, Pigmented villonodular 
synovitis and ankylosing spondylitis for $1 \%$ each (Figure 3 and Table 2).

Table 2. Showing diagnosis.

\begin{tabular}{lllll}
\hline Diagnosis & $\begin{array}{l}\text { Osteoarthriti } \\
\text { s }\end{array}$ & $\begin{array}{l}\text { Rheumatoid } \\
\text { arthritis }\end{array}$ & $\begin{array}{l}\text { Pigmented } \\
\text { villonodular } \\
\text { synovitis }\end{array}$ & $\begin{array}{l}\text { Ankylosing } \\
\text { spondylitis }\end{array}$ \\
\hline No. & 1105 & 34 & 11 & 11 \\
\hline $\begin{array}{l}\text { Percentage } \\
(\%)\end{array}$ & 95.1 & 2.9 & 1 & 1 \\
\hline
\end{tabular}

Among the infected four patients all were diagnosed with osteoarthritis. There was not proven significant statistically of diagnosis for risk factor between infection and non-infection groups. P-value was $0.118(>0.05)$.

Smoking: Out of total patients 216 (18.6\%) were smoker, among male $84.21 \%$ were and among female $2.57 \%$ were smoker. Nine hundred and forty-five $(81.4 \%)$ patients were non-smokers (Table 3).

Table 3. Showing smoking habit between two groups.

\begin{tabular}{llllll}
\hline & \multicolumn{3}{c}{ Smoking } & \multicolumn{1}{c}{ Odd Ratio } & P value \\
\cline { 2 - 3 } Infection (\%) & No & $958(83.7)$ & $187(16.3)$ & & \\
\cline { 2 - 3 } & Yes & $4(25)$ & $12(75)$ & & 0.003 \\
\hline
\end{tabular}

Among sixteen infected TKR patients 12 (75\%) were smokers and 4 was non-smoker (eleven male and one female patients were smokers). Among smokers and non-smokers, smoker patients had increased odds of developing infection 15 times more (odd ratio, 15.37). There was statistically significant between smoking as risk factor and infection, with $\mathrm{p}$ value $0.003(<0.05)$.

Hypertension: Among the total patients 410 patients had HTN, which accounts for $35.3 \%$ and the rests $(64.7 \%)$ had not. In infected group 75\% $(n=12)$ had HTN and rest $25 \%(n=4)$ had not. In non-infected groups 33.7\% $(n=386)$ had HTN and $66.3 \%(n=759)$ had not HTN. Odds of developing infection to be increased between the HTN and non-HTN group (odds ratio, 5.90). Chi square test was performed for significance statistically, but there was not significance proven. $\mathrm{p}$ value was more than $0.05(0.09)$.

Diabetes mellitus: In the study there were $13.1 \%$ patients, who had DM and rest $86.9 \%$ did not had. Among infected group $75 \%$ had DM and remaining $25 \%$ did not had. In noninfected group $12.2 \%$ had and $87.8 \%$ did not have DM.

Table 4. Showing diabetes mellitus between two groups.

\begin{tabular}{llllll}
\hline & \multicolumn{2}{c}{ Diabetes Mellitus } & Odds ratio & p value \\
\cline { 2 - 3 } & No & Yes & & \\
\cline { 1 - 3 } Infection (\%) & No & $1005(87.8)$ & $140(12.2)$ & 21.50 & 0.001 \\
\hline
\end{tabular}

$$
\text { Yes } \quad 4(25) \quad 12(75)
$$

Patients with diabetes mellitus were 21.5 times more likely to develop infection then were those without diabetes. While calculating Chi square test there was significance statistically between infection and diabetes mellitus $(p=0.001)$, which was less than 0.05 (Table 4).

Obesity: Obesity is determined by calculating Body Mass Index (BMI) obtaining by body weight in kilograms divided by squared height in meters. According to WHO a patient who's BMI was $\geq 30 \mathrm{~kg} / \mathrm{m}^{2}$ were noted as obese patients and who were below that as non-obese patients. In the study there were $137(11.8 \%)$ obese $\left(\mathrm{BMI} \geq 30 \mathrm{~kg} / \mathrm{m}^{2}\right)$ patients and they did not have infection. The patients who developed infection were non-obese patients. There was no significance statically between obesity and infection as $\mathrm{p}$ value was $0.78(>0.05$, Table 5).

Table 5. Showing obesity between two groups.

\begin{tabular}{llll}
\hline & \multicolumn{2}{c}{ Obesity } \\
\cline { 2 - 3 } & No & Yes \\
\hline Infection (\%) & No & $1008(88.0)$ & $137(12.0)$ \\
\cline { 2 - 3 } & Yes & $16(100)$ & $0(0.0)$ \\
\hline
\end{tabular}

Malnutrition: Patients categorized in malnutrition who had $\mathrm{BMI}<18.4 \mathrm{~kg} / \mathrm{m}^{2}$, total lymphocyte count $<1,500 / \mathrm{ml}$ and total serum albumin $<3.5 \mathrm{~g} / \mathrm{dL}$. Out of 1161 patients who meet above criteria were fifteen (1.29\%) and those patients did not developed infection. The patients who had adequate nutrition were 1009 (86.91\%) and among them sixteen patients had infection. There was not significance statistically $(\mathrm{p}=0.58)$.

Steroid use: Steroid or immunosuppressive therapy was taken for different purpose; some were for orthopedics related as rheumatoid arthritis, late stage osteoarthritis and some for other systemic causes. Steroid use was defined as any form of systemic steroid therapy for $>1 \mathrm{w}$ in the year before total knee replacement. Among total that meet above criteria were 30 (2.6\%) patients and remaining 1131 (97.4\%) did not had history of steroid use. Out of 30 patients 7 patients had developed infection.

Table 6. Showing use of steroid between two groups.

\begin{tabular}{|c|c|c|c|c|c|}
\hline & & \multicolumn{2}{|l|}{ Steroid use } & \multirow[t]{2}{*}{ Odds ratio } & \multirow[t]{2}{*}{$p$ value } \\
\hline & & No & Yes & & \\
\hline \multirow[t]{2}{*}{ Infection (\%) } & No & $1122(98.0)$ & $23(2.0)$ & 29.1 & 0.00064 \\
\hline & Yes & $9(56.2)$ & $7(43.8)$ & & \\
\hline
\end{tabular}

About infected group $43.8 \%(n=7)$ had history of steroid use and remaining $56.2 \%(n=9)$ did not have history of steroid use. Steroid used patients had increased odds of developing infection 29 times (odds ratio, 29.1) than the patients who did not had history of steroid use. There was association between 
infection and steroid use which shown by Chi square test as significance statically $(\mathrm{p}=0.00064$, Table 6$)$.

\section{Provider related factors}

Surgery time: Surgery time was calculated from start of incision to closure of skin. That was included both single limb and bilateral limb in one setting. This was taken in minutes. The minimum duration was $60 \mathrm{~min}$ and maximum was 245 min (bilateral) with mean $130.90 \mathrm{~min}$.

Table 7. Showing duration of surgery time.

\begin{tabular}{llllll}
\hline & $\begin{array}{l}\text { Minimum } \\
(\mathbf{m i n})\end{array}$ & $\begin{array}{l}\text { Maximum } \\
(\mathbf{m i n})\end{array}$ & Mean $(\mathbf{m i n})$ & SD & p value \\
\hline Overall & 60 & 245 & 130.90 & 40.32 & 0.12 \\
\hline No infection & 60 & 245 & 130.02 & 42.60 & \\
\hline Infection & 135 & 170 & 152.50 & 17.55 & \\
\hline
\end{tabular}

In infected group minimum duration of surgery and mean were higher than non-infected group. Though two groups were not normally distributed so non-parametric test was performed. There was no statistically significance of infection with duration of surgery with $\mathrm{p}$ value of 0.12 , which was greater than 0.05 (Figure 4 and Table 7).

Total blood loss: Total blood loss was calculated as total volume of blood loss during surgery and postoperatively through drains. The mean of blood loss during and after TKR was $751.47 \mathrm{ml}$, with minimum $450 \mathrm{ml}$ and maximum $1150 \mathrm{ml}$. Table below showing blood loss their means and standard deviation on infected and non-infected cases.

Table 8. Showing blood loss between two groups.

\begin{tabular}{llllll}
\hline & $\begin{array}{l}\text { Minimum } \\
(\mathrm{ml})\end{array}$ & $\begin{array}{l}\text { Maximum } \\
(\mathrm{ml})\end{array}$ & Mean $(\mathrm{ml})$ & SD & p value \\
\hline TKR Cases & 450 & 1150 & 751.47 & 157.40 & 0.019 \\
\hline Non-infected & 450 & 1150 & 743.88 & 154.69 & \\
\hline Infected & 800 & 1050 & 937.50 & 110.86 & \\
\hline
\end{tabular}

The mean $(937.50 \mathrm{ml})$ blood loss in infected cases was higher than non-infected cases. There was statistically significance of infection with amount of blood loss with $p$ value of 0.019 which was less than 0.05 . Below box plot also showing their inter quartile range and mean in infected and non-infected groups (Figure 5 and Table 8).

Blood transfusion: Blood transfusion was transfusion of blood during or after surgery. In the study every patient had blood transfusion either intra-operatively or post operatively. Amount of blood transfusion was estimated according to clinical, hemoglobin or blood loss evaluation. The minimal amount of blood transfusion was $200 \mathrm{ml}$ and maximum was $1200 \mathrm{ml}$. with mean of $596.08 \mathrm{ml}$.

In infected group mean amount of blood transfusion was approximately double then non-infected group. There was significance statistically of amount of blood transfusion with infection with $\mathrm{p}$ value of $0.001(<0.05)$. Box plot also showing inter quartile range and mean in two groups (Figure 6 and Table 9).

Table 9. Showing blood transfusion.

\begin{tabular}{llllll}
\hline & $\begin{array}{l}\text { Minimum } \\
(\mathrm{ml})\end{array}$ & $\begin{array}{l}\text { Maximum } \\
(\mathrm{ml})\end{array}$ & Mean $(\mathrm{ml})$ & SD & p value \\
\hline TKR cases & 200 & 1200 & 596.08 & 196.96 & 0.001 \\
\hline Non-infected & 200 & 1000 & 579.59 & 180.46 & \\
\hline Infected & 400 & 1200 & 975 & 163.29 & \\
\hline
\end{tabular}

Duration of hospital stay: Duration of hospital stay counted as pre-operative and post-operative days. Minimum duration of hospital stay was $7 \mathrm{~d}$ and maximum was $38 \mathrm{~d}$ with mean 10.95 d. In infected and non-infected groups the means were $13.35 \mathrm{~d}$ and $10.76 \mathrm{~d}$ respectively, which was higher in infected cases. There was not proven statistically significant with duration of hospital stay and infection with $\mathrm{p}$ value greater than 0.05 $(\mathrm{p}=0.079$, Table 10).

Table 10. Showing duration of hospital stay.

\begin{tabular}{llllll}
\hline & $\begin{array}{l}\text { Minimum } \\
\text { (d) }\end{array}$ & $\begin{array}{l}\text { Maximum } \\
\text { (d) }\end{array}$ & Mean (d) & SD & p value \\
\hline $\begin{array}{l}\text { TKR } \\
\text { cases }\end{array}$ & 7 & 38 & 10.95 & 5.22 & 0.079 \\
\hline $\begin{array}{l}\text { Non- } \\
\text { infection }\end{array}$ & 7 & 38 & 10.76 & 5.16 & \\
\hline Infection & 8 & 30 & 13.35 & 5.05 & \\
\hline
\end{tabular}

In the study all four patients had developed delay infection. Diagnosis of TKR infection was done with help of clinical presentation, radiological evaluation and laboratory parameters. Some results are listed below.

\section{Joint aspirated fluid}

Analysis of knee joint aspirated fluid is one of key diagnostic test for infected TKR. There was mention cut off point for positive for infection if synovial fluid absolute WBC counts of $>1700 / \mathrm{ml}$ and neutrophil percentage $\geq 65 \%$. Out of sixteen infected patients thirteen had positive, which meet above criteria and three patients did not meet those criteria.

\section{Microorganism profile}

Microorganism was culture from joint aspirated fluid and periprosthetic tissue. Different organisms were found among sixteen patients. The organisms causing infection in these patients were Staphylococcus aureus (four patients), Enterobacter cloacle (four patients), Klebsella+Staphylococcus aureus (four patients) and none in four patients (Figure 7). 


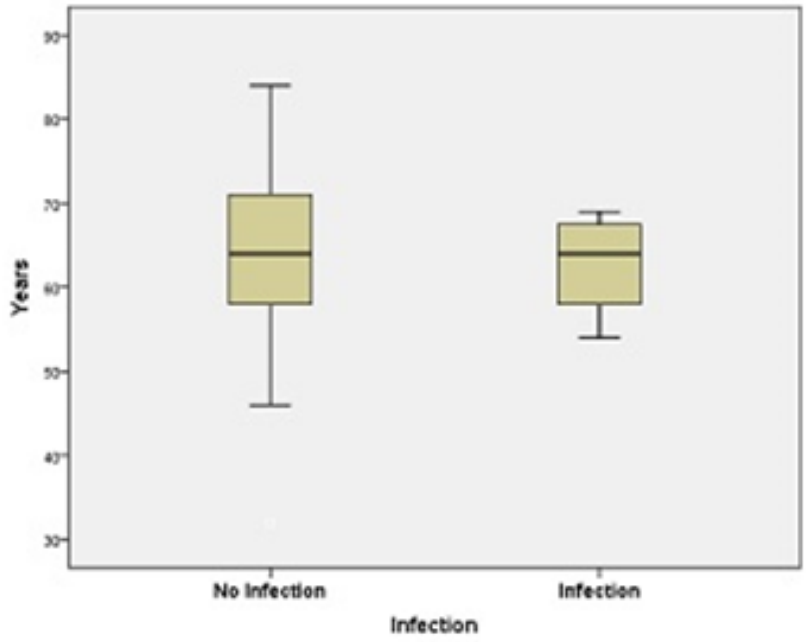

Figure 1. Box plot showing age distribution between two groups.

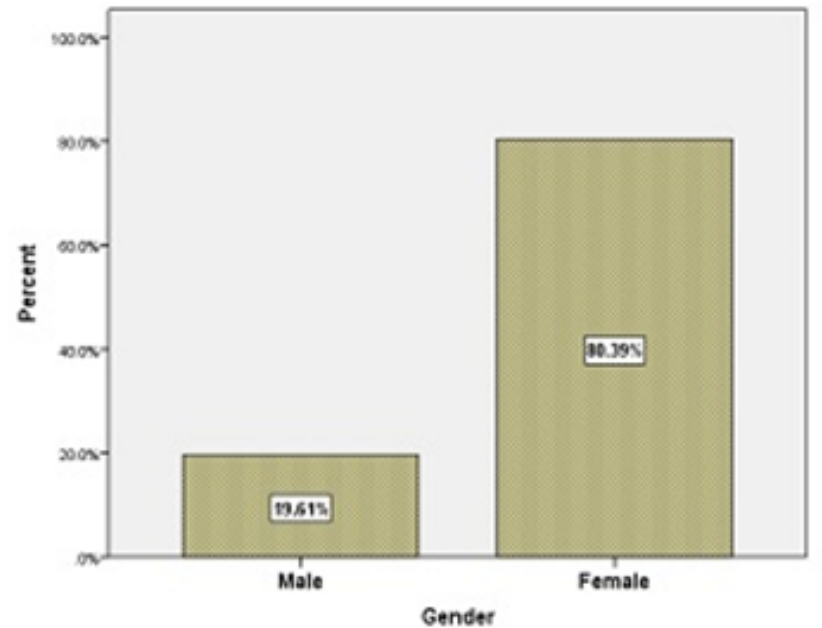

Figure 2. Simple bar graph showing gender distribution.

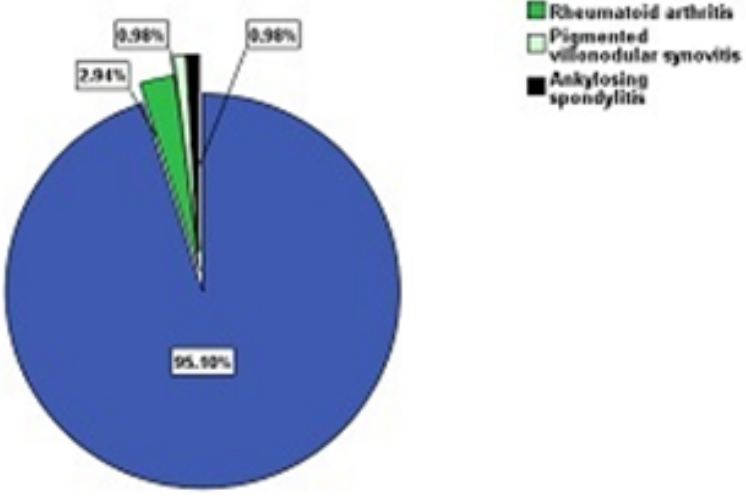

Figure 3. Pie chart showing diagnosis distribution.

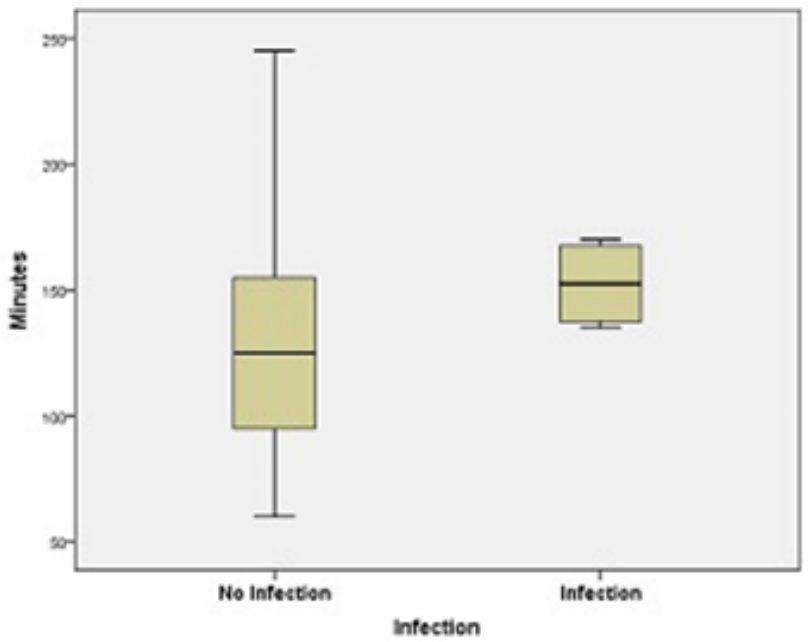

Figure 4. Box plot showing duration of surgery between two groups.

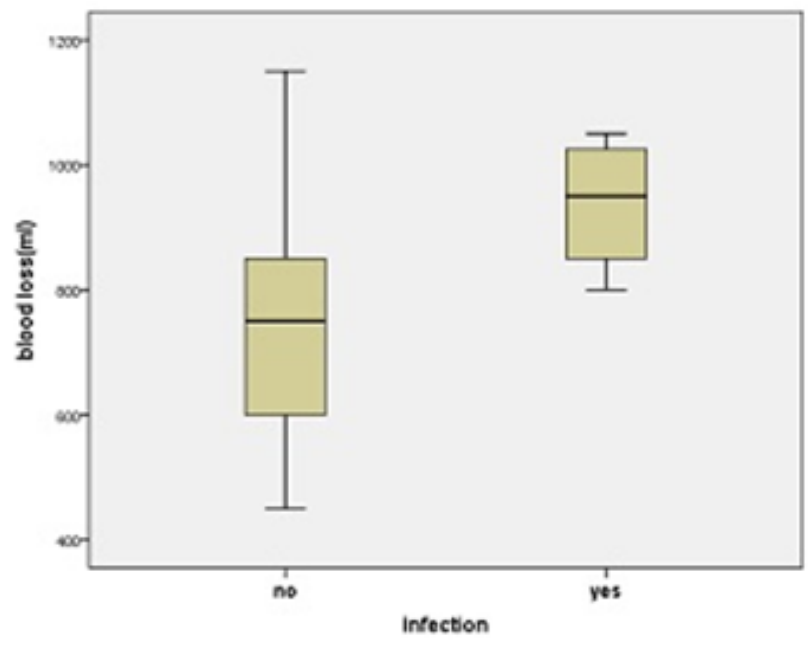

Figure 5. Box plot showing blood loss between two groups.

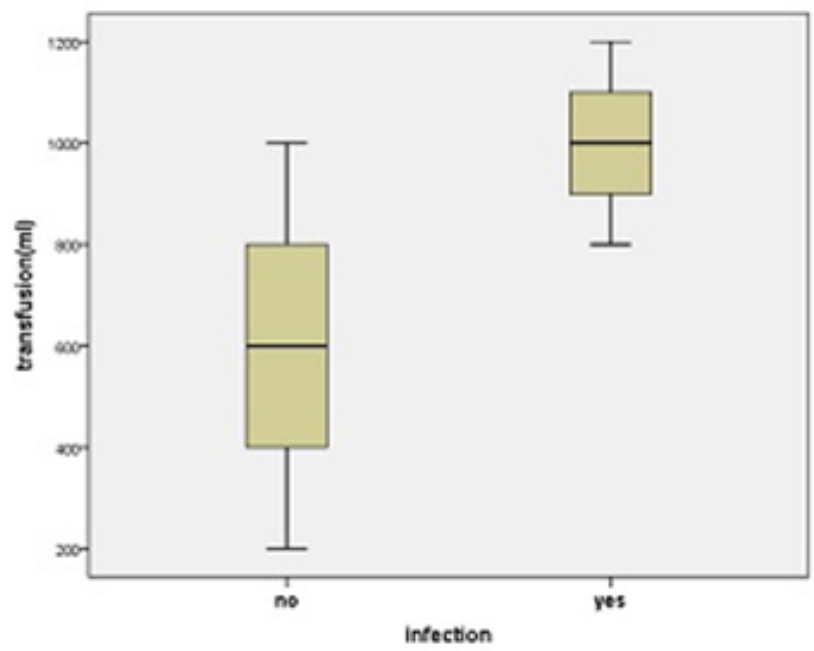

Figure 6. Box plot showing blood transfusion between two groups. 


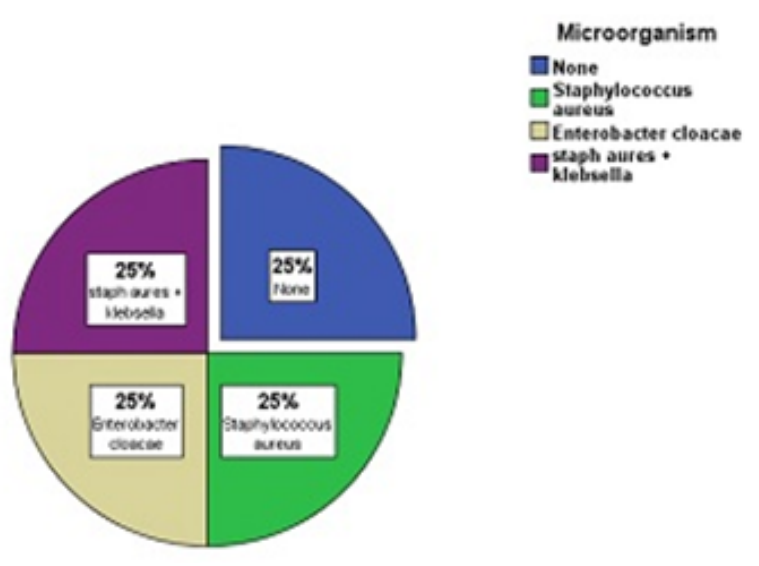

Figure 7. Pie chart showing microorganism profile in cultured joint fluid.

\section{Discussion}

\section{Results analysis}

TKR is among the most common major surgical procedure performed throughout the world nowadays. Demand for TKR for treatment of end stage arthritis is increasing. Historically infection was the most common cause of failure while Gluk describe the first thing prosthesis. Despite continuing efforts to prevent postoperative infections, prosthetic joint infections complicate up to $1 \%$ of primary TKR even in specialized centers [4]. Information on infection incidence in regard to TKR has been analysed from various sources ranging from signal-center studies to large scale multi-institution studies and national registries. No gold-standard definition for TKR infection and no single accepted set of diagnostic criteria are established. Improvement in advance surgical techniques and implant design are continuously being advanced to reduce infection related complications but the numbers of patients suffering PJI are still high. Novel approaches are needed to reduce the burden of infection. So it is wise to know about risk factors which play significance role for infection causation.

In this study primary TKR infection rate was $1.38 \%$, which is a little higher as comparing to literature. Incidence of infected knee replacement in a UK hospital declined from $4.4 \%$ to $1 \%$ after introduction of improved aseptic techniques and routine antibiotic prophylaxis [5]. Some noted PJI rate around $1 \%$ following primary knee replacement in specialized orthopedic setting institution $[4,6]$. In the case of total knee arthroplasty, infection occurred in 0.8-0.9\% of cases in Finland when observed from single institution studies from 1997 to 2006. Similarly, a single institution study in Japan had infection occur in $0.8 \%$ of TKA procedures from 1995 to 2006. Studies in the United States and abroad suggest that infection rates for the general population are similar and are estimated to range from $0.7 \%$ to $1.1 \%[7,8]$. In comparing with that literature infection rate in our study was a little higher despite of improvement in technology and implant design in recent year. This could be due to small sample size and methodology in the study.
In this study mean age of $62.75 \mathrm{y}$ in infected group was slightly lower than that in non-infected group (64.44 y). Age was not found to predispose to infected TKR but in some study it was reported increased risk of infection with increasing age $[9,10]$. This could be due to the dysfunction immune system and other co-morbid conditions. A study performed by Kaye revealed that risk of infection is linearly increasing until age of 65 years then after this age, risk of infection is decreasing by $1.2 \%$ for each additional year [11]. Possible explanation for this phenomenon could be that unhealthy old patients are less frequently treated by surgical interventions than their healthy peers and also could be resulted from selection of a relatively healthy group among older patients. In our study despite of female and male ratio of $4.1: 1$, there were nearly equal numbers of infection in both sexes that could be possibility of higher infection rate in male sex than female although no significantly statistical difference. Further study with adequate sampling size need to be carried out on this topic to show significance between two sexes.

In our study there was no significance association between infection and diagnosis of disease especially rheumatoid arthritis. Indications for TKR were osteoarthritis in all four infected cases. Some study compared patient with rheumatoid arthritis have 2 to 4 fold greater risk of acquiring postoperative infection rate than patient with primary osteoarthritis [12-14]. In some study the postoperative infection rates for rheumatoid arthritis report a range from $2.2 \%$ to $9.9 \%$. Infection occurs fairly soon after operation in OA patients but in RA infection is late presentation believed to be hematogenous spread. RA patients are frequently treated with complex drug regimes that include NSAIDs, corticosteroids, methotrexate, DMARDs, and other biologic agents. All of which have direct or indirect influence on wound healing and potential risk of infection. In our study number of RA cases were small, which could be because nowadays RA patients are treated with newly investigated drugs with more effectiveness than surgically. Adequate sample from RA cases are needed to address this issue. In the study patients who used steroids were for different purpose, orthopedics related or other systemic cause, which accounts for $10.8 \%$, among which $75 \%$ had history of steroid use in infected group. That also has shown significance association with infection $(\mathrm{p} \leq 0.05)$, odds ratio 33.75 against non-infected group. Some studies in related topics reported that use of oral steroid has been shown to increase the risk of infection in total knee replacement in univariate analysis $[15,16]$ but other studies revealed no effect of corticosteroids on infected knee replacement [17,18]. Steroid is antiinflammatory medication which suppress host's defense against infection and decrease host's inflammatory response. Nevertheless, special cautions need to take the patients who are on steroids. It is advised to stop steroid use couple of week before elective TKR to overcome risk of infection. Intraarticular injections of corticosteroids have been used for the treatment of osteoarthritis of the knee since many years ago by orthopedic surgeons, general practitioners and rheumatologists but which has little evidence to support any lasting beneficial value and its risk towards the infection. 
In our study $18.6 \%$ were smoker among infected cases $75 \%$, showing significance statistically with $\mathrm{p}$ value of 0.003 and odds ratio 15.37. There were studies which show smoking is a significant risk factor for intra-operative and postoperative morbidity $[19,20]$. Smoking impair in bone metabolism, fracture repair and in increasing the rate of postoperative infection and incidence of non-union [21]. Tobacco consumption is a very important risk factor for serious postsurgical complications. Tobacco use may delays primary wound healing and may increase the risk of infection. Nicotine inhibits the proliferation of Red Blood Cells (RBC), macrophages and fibroblasts, which all present in order to heal injured tissue. Nicotine has also effect on increased platelet adhesiveness, which causes micro-clots and decreases microperfusion. This eventually leads to clot formation and reduced blood flow. Nicotine produces cutaneous vasoconstriction because of the release of catecholamine. So from above studies it is proven that smoking has significance effect on infection. Hence smoking can be potentially modifiable to promote patients general health, intervention should be made by counselling nicotine replacement therapy, smoking reduction either smoking cessation.

In the study there were $14.7 \%$ DM patients, among that in the infected group $75 \%$ had DM. Which show significance statistically with $p$ value of 0.001 and odds ratio 21.50 . Studies show that diabetes has been associated with an increased risk of infection in all orthopedics fields [22,23]. Up to $10 \%$ of patients undergoing knee replacement have diabetes [24-26]. Some case series studies reports infection rate of up to $7 \%$ $[27,28]$ and in case-control report significantly more knee replacement infections have been observed with diabetes cases [6,15,29]. One study in 2011 revealed that, in infected group there were significantly more diabetes patients then in noninfected group of lower limb arthroplasty (22\%). Significantly higher perioperative blood glucose level was found in infected patients. Risk for the infection is increased more than two-fold if postoperative morning hyperglycemia (BG>200 mg/dl). On first postoperative day if their morning blood glucose level was $>140 \mathrm{mg} / \mathrm{dl}$, non-diabetic patients were three times more likely to develop the infection [30]. It is not clear which factors contribute to the occurrence of joint replacement infection in diabetic patient. Infection have been found to be strongly associated with use of insulin but it is unclear whether insulin therapy predispose to infection or if it acts as a surrogate marker for the severity of disease. Other possible causative factors include the oral anti-diabetic treatment, diabetes related complications (atherosclerosis of peripheral arteries, peripheral neuropathy and diabetic ulcer) and other severe glucose metabolism disorder.

In the study there were only $13.09 \%$ of patients who had obese and malnutrition all those were in non-infected group. The prevalence of obesity is increasing nowadays through worldwide. Studies show that, over half of total joint recipients were obese $[31,32]$. Several studies shown that obesity represents a risk factor for infection in joint replacement surgery [33-36]. Namba performed a study in 2005 , which demonstrated that the risk of an infection was 6.7 times higher in obese patients who underwent total knee replacement. In number of other studies no statistically significant difference has been observed $[3,15,17,37,38]$. In obese patients causes of infection believe to be increased frequency of hematoma development and prolonged drainage due to greater extent of surgical dissection, fat tissue hypoperfusion with decreased tissue oxygenation and reduced oxygen tension with consequent decreased oxidative killing potential of neutrophils against pathogens, low tissue levels of prophylactic antibiotics due to improper dose adjustment to weight. About malnutrition studies shown that poor nutritional status is a well-known risk factor for deep infection after orthopedic surgical procedure [39]. Infection rate is high has been observed in malnutrition [6,15]. Our data probably could not support because of small sample size or classification and definition of obesity and malnutrition as the same as western people despite of different in physical status in Chinese people. It is possible to reduce the risk of infection for patients in this group by controlling weight, applying an appropriate dose of prophylactic antibiotics. If there is evidence of malnutrition in patient undergoing elective orthopedic procedure, preoperative and postoperative nutritional support should be provided. The main aim should be to increase the total serum albumin level, improve the total lymphocyte count and increase total serum transferring levels which positively influence wound healing potential and consequently reduce the risk of infection. During perioperative period patients should obtain sufficient dietary nutrition or protein, vitamin and mineral supplementation.

In our study the mean duration of surgery was $130.90 \mathrm{~min}$ and in infected group mean duration was $152.50 \mathrm{~min}$. There was no significance between surgery duration and infection. However, studies showing that duration of surgery has been associated with infected knee replacement both in adjusted analysis $[40,41]$ and univariate analysis $[6,18]$ which is included in the NNIS risk index. A study between 1993 and 1999 among 6489 patients, who underwent TKR, assessed the association between the duration of the surgery and the risk of postoperative infection. 104 infected patients were matched with 236 controls group. Which shown that patients who do not have infections $(n=236)$ had surgery durations of $94 \pm 28$ min, and patients who had infection $(n=104)$ had durations of surgery $127 \pm 45 \min (p<0.001)$ [42]. This revealed that there is significance association between duration of surgery and infection risk in TKR. Probably in our study we included unilateral knee and bilateral knee replacement together and did not set the cut-off point of 2-2.5 h as risk of infection, which usually taken. It was reported that after cut-off point (2-2.5 h) the infection rate increased significantly.

There were higher amounts of blood loss and blood transfusion in infected group than non-infected group of the study. The mean of blood loss during and after TKR was $743.88 \mathrm{ml}$ in non-infected group while in infected group the mean blood loss was $937.50 \mathrm{ml}$. Similarly, mean amount of blood transfusion was $579.59 \mathrm{ml}$ in non-infected group while in infected group mean amount of blood transfusion was $975 \mathrm{ml}$, approximately double then non-infected group. Some studies report that blood loss in routine TKR is modest and does not seem alone to 
predispose to infection $[16,18,43]$. However, higher rates of infection have also been reported when blood transfusions are required [16], which is particularly true for allogenous blood transfusion $[15,16]$. In blood there are lots of components which act immunologically to overcome infection if much blood lost there were lack of those components and patient prone to infection. Frequently patients are found to have a lower than anticipated post-operative haemoglobin due to extravasation of blood into the tissues, loss due to haemolysis and residual blood in the knee, all of which are hidden. Attempts to reduce post-operative blood loss are encouraged to avoid the risks of blood loss and transfusion-related complications. To reduce blood loss after TKR pharmacological measures include topically applied fibrin sprays and the intravenous administration of tranexamic acid are applied. Allogenic blood transfusion is also associated with transfusion complications as immunological and other noninfectious.

\section{Future considerations}

Until now the decline in PJI rate has been achieved by improving the (extrinsic factors) operating environment, surgical techniques and introducing antibiotic prophylaxis. But future ways to consider about patients intrinsic factors which have directly effect on outcome. Results motivate controlling patients' general health and co-morbid conditions preoperatively. Multicenter prospective RCT study with large amount of data will be needed in further studies on this field to allow adequate statistical analysis. Arthroplasty Registry will consider for potential source of all valuable comparative data. In this study nearly $15.5 \%$ had lost follow up because of changed their contact address, which should been addressed in clinical practice in order to carry out a study that can produce accurate information in future.

\section{Limitations of study}

The major drawback of this study is small sample size and all data is restricted to one single hospital and too many patients lost to follow up because of change in patient contact details such as address or phone number. Besides that study was retrospective, required data rely on by others recorded data which were not sufficient for analysis.

\section{References}

1. Robertsson O, Knutson K, Lewold S. The Swedish knee arthroplasty register 1975-1997: an update with special emphasis on 41,223 knees operated on in 1988-1997. Acta Orthopaedica 2001; 72: 503-513.

2. Rantanen P, Keinonen A, Makela A. The 2004 implant yearbook on orthopaedic endoprostheses. Finnish Arthroplasty Register. Publ Nat Agency Med 2006.

3. Chesney D, Sales J, Elton R. Infection after knee arthroplasty: a prospective study of 1509 cases. J Arthroplast 2008; 23: 355-359.
4. Phillips J, Crane T, Noy M. The incidence of deep prosthetic infections in a specialist orthopaedic hospital a 15-year prospective survey. J Bone Joint Surg Br 2006; 88: 943-948.

5. Blom AW, Brown J, Taylor AH. Infection after total knee arthroplasty. J Bone Joint Surg Br 2004; 86: 688-691.

6. Peersman G, Laskin R, Davis J. Infection in total knee replacement: a retrospective review of 6489 total knee replacements. Clin Orthop Relat Res 2001; 392: 15-23.

7. Kurtz S, Ong K, Lau E. Projections of primary and revision hip and knee arthroplasty in the United States from 2005 to 2030. J Bone Joint Surg Am 2007; 89: 780-785.

8. Parvizi J, Adeli B, Zmistowski B. Management of periprosthetic joint infection: the current knowledge:AAOS exhibit selection. J Bone Joint Surg 2012; 94: 104.

9. Delgado-Rodríguez M, Gomez-Ortega A, Sillero-Arenas M. Epidemiology of surgical-site infections diagnosed after hospital discharge: a prospective cohort study. Infect Contr Hosp Epidemiol 2001; 22: 24-30.

10. Scott JD, Forrest A, Feuerstein S. Factors associated with postoperative infection. Infect Contr Hosp Epidemiol 2001; 22: 347-351.

11. Kaye KS, Schmit K, Pieper C. The effect of increasing age on the risk of surgical site infection. J Infect Dis 2005; 191: 1056-1062.

12. Moucha CS, Clyburn T, Evans RP. Modifiable risk factors for surgical site infection. J Bone Joint Surg 2011; 93: 398-404.

13. Jamsen E, Huhtala H, Puolakka T. Risk factors for infection after knee arthroplasty-a register-based analysis of 43,149 cases. J Bone Joint Surg 2009; 91: 38-47.

14. Schrama JC, Espehaug B, Hallan G. Risk of revision for infection in primary total hip and knee arthroplasty in patients with rheumatoid arthritis compared with osteoarthritis: a prospective, populationbased study on 108,786 hip and knee joint arthroplasties from the Norwegian Arthroplasty Register. Arthritis Care Res 2010; 62: 473-479.

15. Berbari EF, Hanssen AD, Duffy MC. Risk factors for prosthetic joint infection: case-control study. Clin Infect Dis 1998; 27: 1247-1254.

16. Parvizi J, Ghanem E, Joshi A. Does excessive anticoagulation predispose to periprosthetic infection? J Arthroplast 2007; 22: 24-28.

17. Lai K, Bohm ER, Burnell C. Presence of medical comorbidities in patients with infected primary hip or knee arthroplasties. J Arthroplast 2007; 22: 651-656.

18. Minnema B, Vearncombe M, Augustin A. Risk factors for surgical-site infection following primary total knee arthroplasty. Infect Contr Hosp Epidemiol 2004; 25: 477-480.

19. Pearce A, Jones R. Smoking and anesthesia: preoperative abstinence and perioperative morbidity. Anesthesiology 1984; 61: 576-584. 
20. Moller A, Tonnesen H. Risk reduction: perioperative smoking intervention. Best Pract Res Clin Anaesthesiol 2006; 20: 237-248

21. Kwiatkowski TC, Hanley EN, Ramp WK. Cigarette smoking and its orthopedic consequences. Am J Orthop Belle Mead NJ 1996; 25: 590-597.

22. Jamsen E, Nevalainen P, Kalliovalkama J. Preoperative hyperglycemia predicts infected total knee replacement. Eur J Int Med 2010; 21: 196-201.

23. Edmonston DL, Foulkes GD. Infection rate and risk factor analysis in an orthopaedic ambulatory surgical center. J Surg Orthop Adv 2010; 19: 174-176.

24. Meding JB, Reddleman K, Keating ME. Total knee replacement in patients with diabetes mellitus. Clin Orthop Relat Res 2003; 416: 208-216.

25. Meding JB, Klay M, Healy A. The prescreening history and physical in elective total joint arthroplasty. J Arthroplast 2007; 22: 21-23.

26. Papagelopoulos PJ, Idusuyi OB, Wallrichs SL. Long term outcome and survivorship analysis of primary total knee arthroplasty in patients with diabetes mellitus. Clin Orthop Relat Res 1996; 330: 124-132.

27. Yang K, Yeo S, Lee BP. Total knee arthroplasty in diabetic patients: a study of 109 consecutive cases. J Arthroplast 2001; 16: 102-106.

28. England SP, Stern SH, Insall JN. Total knee arthroplasty in diabetes mellitus. Clin Orthop Relat Res 1990; 260: 130-134.

29. Syahrizal A, Kareem B, Anbanadan S. Risk factors for infection in total knee replacement surgery at hospital Kuala Lumpur. Med J Malay 2001; 56: 5-8.

30. Mraovic B, Suh D, Jacovides C. Perioperative hyperglycemia and postoperative infection after lower limb arthroplasty. J Diab Sci Technol 2011; 5: 412-418.

31. Namba RS, Paxton L, Fithian DC. Obesity and perioperative morbidity in total hip and total knee arthroplasty patients. J Arthroplast 2005; 20: 46-50.

32. Fehring TK, Odum SM, Griffin WL. The obesity epidemic: its effect on total joint arthroplasty. J Arthroplast 2007; 22: 71-76.

33. Dowsey MM, Choong PF. Obesity is a major risk factor for prosthetic infection after primary hip arthroplasty. Clin Orthop Relat Res 2008; 466: 153-158.
34. Lubbeke A, Moons KG, Garavaglia G. Outcomes of obese and nonobese patients undergoing revision total hip arthroplasty. Arthritis Care Res 2008; 59: 738-745.

35. Dowsey MM, Choong PF. Obese diabetic patients are at substantial risk for deep infection after primary TKA. Clin Orthop Relat Res 2009; 467: 1577-1581.

36. Samson AJ, Mercer GE, Campbell DG. Total knee replacement in the morbidly obese: a literature review. ANZ J Surg 2010; 80: 595-599.

37. Spicer D, Pomeroy D, Badenhausen W. Body mass index as a predictor of outcome in total knee replacement. Int Orthop 2001; 25: 246-249.

38. Foran JR, Mont MA, Etienne G. The outcome of total knee arthroplasty in obese patients. J Bone Joint Surg 2004; 86 : 1609-1615.

39. Evans RP. Surgical site infection prevention and control: an emerging paradigm. The J Bone Joint Surg 2009; 91: 2-9.

40. De Boer A, Geubbels E, Wille J. Risk assessment for surgical site infections following total hip and total knee prostheses. J Chemother 2001; 13: 42-47.

41. Gastmeier P, Sohr D, Brandt C. Reduction of orthopaedic wound infections in 21 hospitals. Arc Orthop Trauma Surg 2005; 125: 526-530.

42. Peersman G, Laskin R, Davis J. Prolonged operative time correlates with increased infection rate after total knee arthroplasty. HSS J 2006; 2: 70-72.

43. Saleh K, Olson M, Resig S. Predictors of wound infection in hip and knee joint replacement: results from a 20 year surveillance program. J Orthop Res 2002; 20: 506-515.

\section{*Correspondence to}

Yumin Zhang

Joint Surgery Department

Hip Ward

Hong-Hui Hospital

Xi'an Jiaotong University College of Medicine

PR China 